\title{
Neuroradiologische Befunde bei der Hemiatrophia facialis progressiva (Parry-Romberg-Syndrom)
}

Die Hemiatrophia facialis progressiva wurde 1825 erstmals von Parry und 1846 von Romberg beschrieben. Es handelt sich um eine meist einseitige, langsam progrediente, aber selbst limitierende Atrophie der Gesichtsweichteile mit Bevorzugung des Versorgungsgebietes des N. trigeminus (Rogers BO et al, Int Congress Series 1963; 66: 681). Die Erkrankung beginnt bei vielen Patienten im ersten oder zweiten Dezennium mit einer bläulich-bräunlichen Hyperpigmentierung der Haut über der Augenbraue oder unter dem Unterlid. Ipsilateral folgen andere typische Symptome wie der Verlust von Wimpern oder der Augenbraue, ein Pigmentverlust der Haare, eine Alopezie oder eine linienförmige Hautvertiefung (en coup de sabre). Die Atrophie betrifft in erster Linie die Gesichtshaut und das subkutane Gewebe. Nur wenn die Krankheit im ersten Lebensjahrzehnt beginnt, ist eine Atrophie des Gesichtsschädels zu befürchten. Die Gegenseite oder die Arme werden nur selten bei einem weit fortgeschrittenen Krankheitsbild erfasst. Häufiger wird eine ipsilaterale zerebrale Beteiligung gefunden, deren genaue Inzidenz jedoch nicht bekannt ist. Neurologische Symptome, die man als eine Trias aus Herdsymptomen, psychischen Veränderungen und vegetativen Störungen zusammenfassen kann, treten bei 10-15\% der Patienten auf (Terstegge $\mathrm{K}$ et $\mathrm{al}$, Radiologe 1993; 33: 585). Sie können auch dann noch zunehmen, wenn die Atrophie der Gesichtsweichteile bereits zum Stillstand gekommen ist (Asher SW et al, Arch Neurol 1982; 39: 44). Bei $10-$ $40 \%$ der Patienten wird die Hemiatrophie von Augenerkrankungen begleitet.

Histologisch findet man eine unspezifische chronische Entzündung mit perivaskulärer lymphozytärer Infiltration und Atrophie von Haut und Haarfollikeln. Die elastischen Fasern bleiben im Gegensatz zur Sklerodermie erhalten. Eine intrakranielle Beteiligung ist durch

Fortschr Röntgenstr 2000; 172: 785-787 (C) Georg Thieme Verlag Stuttgart · New York ISSN 1438-9029 eine einseitige subakute Meningoenzephalitis mit leptomeningealer Fibrose und lymphozytären Infiltraten gekennzeichnet. Zerebral wurden verkalkende Gefäßmalformationen, Marklagergliosen von Groß- und Kleinhirn sowie dysmorphe Sulci beschrieben.

Die Ätiologie der wahrscheinlich nicht erblich bedingten Erkrankung mit weiblicher Prädominanz (Frauen: Männer = 3:2) konnte bisher nicht abschließend geklärt werden. Als Ursachen werden ein chronisch entzündlicher Prozess (Cory RC et al, MNR 1997; 18: 751), möglicherweise in Verbindung mit einer sympathischen Dysfunktion, eine Störung des rostralen Neuralrohres (Schievink WI et al, Neurosurgery 1996; 38: 1237) oder ein neurokutanes Syndrom diskutiert. Die Behandlungsmöglichkeiten des Parry-Romberg-Syndroms sind begrenzt. Die Wirksamkeit einer immunsuppressiven Therapie konnte bisher nicht gesichert werden. Auch die Ergebnisse nach plastischer Rekonstruktion oder Sympathikolyse waren unterschiedlich (Rogers BO et al, Int Congress Series 1963; 66: 681).

\section{Fallbeschreibung}

Unsere Patientin (Abb.1) kam im Alter von 38 Jahren nach einem erstmaligen zerebralen Anfall zur stationären Aufnahme. Die Lichtreaktion der entrundeten rechten Pupille war verzögert. Der übrige neurologische Befund war unauffällig. Im Kindesalter war eine Verdickung der frontalen Kopfhaut rechts aufgefallen, die sich in Richtung auf die rechte Augenbraue ausdehnte und als linienförmige Hautvertiefung ausheilte. Andere wesentliche Vorerkrankungen bestanden nicht.

In der kranialen CT fanden wir neben der frontalen Atrophie der extrakraniellen Weichteile rechts ipsilaterale Verkalkungen im parietalen Kortex, in der darüberliegenden Hirnhaut und im frontalen Marklager (Abb. 2). Parietal waren das angrenzende Marklager und Teile der Capsula externa, frontal das periventrikuläre Marklager dichtegemindert. Kontralateral wurden keine Veränderungen

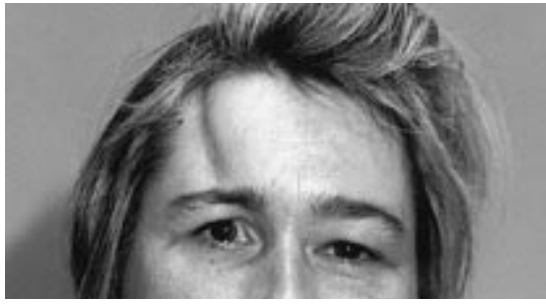

Abb. 1 38-jährige Patientin mit einer für das Parry-Romberg-Syndrom typischen Hautvertiefung über der rechten Stirn und einer Retraktion des rechten Augenlides. Der laterale Anteil der rechten Augenbraue ist gelichtet. Ipsilaterale Pigmentstörung der entrundeten Iris und Enophthalmus.

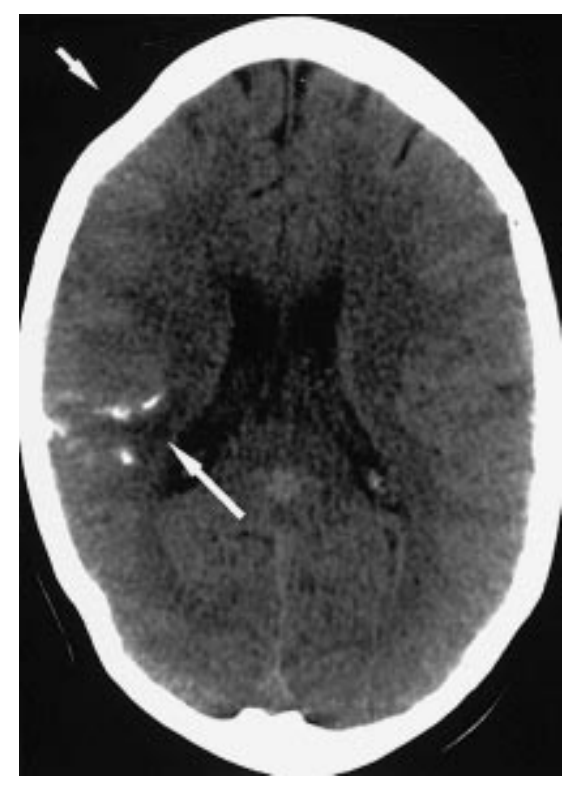

Abb. 2 Die kraniale CT zeigt eine Verkalkung des parietalen Kortex. Das angrenzende Marklager ist dichtegemindert (langer Pfeil). Einziehung der Haut über der rechten Stirn (kurzer Pfeil) bei regelrechtem knöchernen Befund im Knochenfenster (nicht abgebildet).

gefunden, ossäre Veränderungen waren nicht erkennbar.

Neben der Atrophie der frontalen Subkutis rechts (Abb. 3 u. 4) fiel MR-tomographisch ein gleichseitiger Enophthalmus infolge einer Volumenminderung des retrobulbären Fettkörpers auf. Der parietale Kortex war verdickt (Abb. 3 u. 4). In den angrenzenden, fast ganz verstrichenen Sulci fanden wir eine pathologisch vermehrte Kontrastmittelanreicherung (Abb. 4). Ob es sich hierbei um eine leptomeningeale oder venöse Anreicherung handelt, war nicht zu entscheiden. Die in der CT dichtegemin- 


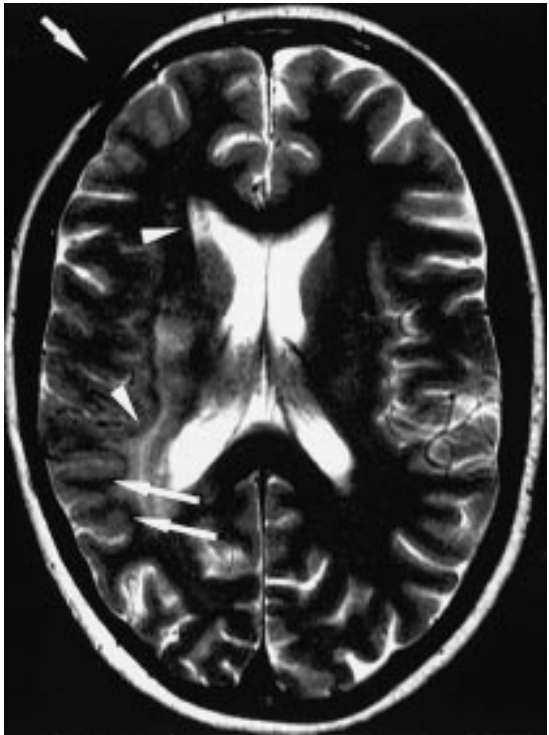

Abb. 3 Die flächigen und fleckigen Signalsteigerungen des rechtsseitigen Marklagers in der $\mathrm{T}_{2}$-Gewichtung (Pfeilspitzen) sind Zeichen einer Marklagergliose und eines periventrikulären Substanzdefektes. Der parietale Kortex ist rechts verdickt (lange Pfeile). Die Sulci sind hier z. T. verstrichen. Rechts frontal ist die Subkutis atrophiert (kurzer Pfeil).

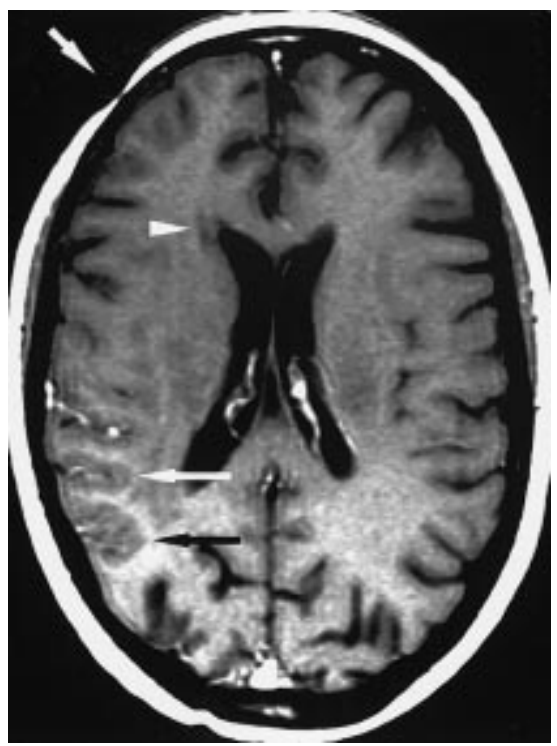

Abb.4 In der $\mathrm{T}_{1}$-Gewichtung hypointenses Signal des periventrikulären Substanzdefektes (Pfeilspitze). Die Marklagergliose ist nicht erkennbar. Der parietale Kortex ist verdickt (lange Pfeile). Venöse oder leptomeningeale Kontrastmittelanreicherung der dazwischen liegenden engen Sulci. Atrophie der rechts frontalen Subkutis (kurzer Pfeil). derten Areale des Marklagers zeigten in der $\mathrm{T}_{2}$-Gewichtung ein hyperintenses Signal (Abb. 3) mit zum Teil hypointensem Korrelat in der $\mathrm{T}_{1}$-Gewichtung (Abb.4) und ohne pathologische Kontrastmittelanreicherung. Zusätzlich wurden fleckige Signalsteigerungen im Putamen und in der Capsula externa gefunden. Bei rechts temporobasal gelegenen liquorisointensen Veränderungen handelt es sich wahrscheinlich um Virchow-Robin-Räume oder Liquorzysten.

\section{Diskussion}

Zusätzlich zu der bei unserer Patientin durch CT und MRT nachgewiesenen einseitigen Atrophie von subkutanem Fettgewebe und retrobulbärem Fettkörper werden in der Literatur weitere extrakranielle Veränderungen beschrieben. Dazu gehören eine Atrophie des Augapfels (Asher SW et al, Arch Neurol 1982; 39: 44), eine Verdickung der äußeren Augenmuskeln, eine Verdichtung des retrobulbären Fettkörpers in der CT und eine einseitige Atrophie der Gesichtsmuskeln. Von einer fokalen Ausdünnung der Schädelkalotte oder Verschmächtigung der Nasennebenhöhlen (Schievink WI et al, Neurosurgery 1996; 38: 1237) blieb unsere Patientin verschont. Die in der Literatur beschriebene fokale Dysmorphie von Kortex und Sulci mit pathologisch vermehrter meningealer oder kortikaler Kontrastmittelanreicherung entspricht unseren CT-Befunden ebenso wie die klein- bis grobfleckigen oder linienförmigen Verkalkungen und die umschriebenen Dichteminderungen von grauer und weißer Substanz (Terstegge K et al, Radiologe 1993; 33: 585). Die entsprechenden MR-Befunde eines fokal verplumpten Oberflächenreliefs mit verdickten Gyri, schlecht abgrenzbaren Sulci und einer vermehrten leptomeningealen Kontrastmittelanreicherung stimmen ebenfalls mit den Literaturangaben überein. Eine verminderte Abgrenzbarkeit des Rindenbandes lag bei unserer Patientin nicht vor. Eine kortikale Kontrastmittelanreicherung kann die Abgrenzbarkeit der Meningen erschweren. Wie bei unserer Patientin sind die zerebralen Verkalkungen in der $\mathrm{T}_{2}$-Gewichtung meist hyper- und in der $\mathrm{T}_{1}$-Gewichtung hypointens. Es wurden aber auch Verkalkungen mit einem hypointensen Signal in der $\mathrm{T}_{2}$-Gewichtung beschrieben. Die fleckige Signalsteigerung der weißen Substanz oder der Basalganglien in der $\mathrm{T}_{2}$-Gewichtung mit zum Teil hypointensem Korrelat in der
$\mathrm{T}_{1}$-Gewichtung ist ein weiterer typischer Befund der progredienten fazialen Hemiatrophie. Die Herde können an Größe zunehmen und vorübergehend Kontrastmittel aufnehmen. Wir fanden MRtomographisch keine Störung der BlutHirn-Schranke. Auch intraaxiale zystische Veränderungen wurden bereits beschrieben. Diese betrafen jedoch die Basalganglien und nicht wie bei unserer Patientin den basalen Temporallappen. Eine einseitige Hirnvolumenminderung bestand bei unserer Patientin nicht. Berichte einer kontralateralen oder beidseitigen Hirnvolumenminderung sollten mit Zurückhaltung gewertet werden: Bei vielen dieser Patienten gab es lediglich eine Röntgenaufnahme des Schädels oder eine Pneumenzephalographie. In einem Fall wurde eine unspezifische Dichteanhebung in der kranialen CT als Hinweis auf eine kontralaterale Beteiligung gewertet. Eine von der Hemiatrophie unabhängige generalisierte Hirnvolumenminderung wurde nicht diskutiert. Während die ToF-Angiographie unserer Patientin einen regelrechten Befund zeigte, wurden bei anderen Patienten ipsilateral zur Hemiatrophie gelegene Hypoplasien der hirnversorgenden Arterien, arteriovenöse Fisteln und multiple, bilaterale Aneurysmen beschrieben.

Tab.1 Typische Befunde der Hemiatrophia facialis progressiva.

- Hemiatrophie der Gesichtsweichteile

- Kortikale Dysmorphie und leptomeningeale KM-Anreicherung

- Verkalkungen und Substanzdefekte von grauer und weißer Substanz

Die Ätiologie des Parry-Romberg-Syndroms kann auch mit den von uns erhobenen Befunden nicht geklärt werden. Die zerebralen Veränderungen passen zu einer fokalen Enzephalitis, die Anamnese ist in unserem Fall für den von anderen Autoren beschriebenen schubweise chronisch progredienten Verlauf jedoch untypisch. Für eine einseitige Irritation des Sympathicus als Ursache der Erkrankung sprechen die Übereinstimmung des Verteilungsmusters der kutanen und zerebralen Veränderungen mit dem Versorgungsbereich der sympathischen Bahnen aus dem Ganglion cervicale superius, einzelne Fallberichte und tierexperimentelle Untersuchungen. 
Für Patienten, die an einem Parry-Rom- immunsuppressiv behandelt werden berg-Syndrom leiden, liegt die Bedeu- sollten.

tung der neuroradiologischen Diagnostik im frühzeitigen Nachweis akut entzündlicher ZNS-Veränderungen, die

K. Kirchhof, T. Welzel, Heidelberg

K. Ziegler, Flensburg 